Kernaussagen

- Im mittleren Lebensalter ist die gesundheitliche Ungleichheit stark ausgeprägt, was sich unter anderem an einem höheren Erkrankungs- und vorzeitigen Sterberisiko bei Personen mit niedrigem Sozialstatus festmachen lässt.

- In den ersten Jahren nach dem Übergang in den Ruhestand besteht die gesundheitliche Ungleichheit fort, zum Teil allerdings in abgeschwächter Form.

- Im weiteren Altersgang kommt es mitunter zu einer weiteren Verringerung der gesundheitlichen Ungleichheit, wobei allerdings sehr unterschiedliche Verlaufsmuster je nach betrachtetem Gesundheitsaspekt zu beobachten sind.

- Bei Männern ist eher als bei Frauen eine Verringerung der gesundheitlichen Ungleichheit festzustellen, was unter anderem mit der höheren vorzeitigen Sterblichkeit, die Männer mit niedrigem Sozialstatus verstärkt betrifft, zusammenhängen dürfte.

- Im Zuge des demografischen Wandels könnte es aufgrund des Rückgangs der vorzeitigen Sterblichkeit auch bei Männern zu einer größeren Stabilität der gesundheitlichen Ungleichheit bis ins hohe Alter bzw. zu einer späteren Annäherung zwischen den Statusgruppen kommen.

1/2016 7. Jahrgang

\section{Gesundheitliche Ungleichheit im höheren Lebensalter}

Soziale Unterschiede in der Gesundheit und Lebenserwartung stellen ein zentrales Thema von Public Health und Gesundheitspolitik dar. Festgemacht werden die sozialen Unterschiede häufig am sozialen Status, der die Stellung von Personen bzw. Haushalten auf der »sozialen Stufenleiter « beschreibt und in empirischen Untersuchungen zumeist über Angaben zu Bildung, Beruf und Einkommen ermittelt wird (Hradil 2001, Galobardes et al. 2006, Lampert, Kroll 2009, Lampert et al. 2013b). Inzwischen weist eine Vielzahl an Studien darauf hin, dass Personen mit niedrigem sozialen Status weitaus häufiger als Personen mit mittlerem und höherem Status von chronischen Erkrankungen und Beschwerden, Behinderungen und Funktionseinschränkungen sowie aus diesen resultierenden Beeinträchtigungen der Lebensqualität und sozialen Teilhabe betroffen sind (Mielck 2000, Marmot, Wilkinson 2006, Siegrist, Marmot 2006, Richter, Hurrelmann 2009, European Commission 2013, Lampert et al. 2013a).

Die Gründe hierfür sind vielfältig und unter anderem in Unterschieden zwischen den Statusgruppen in Bezug auf den finanziellen Handlungsspielraum und materiellen Lebensstandard, die Wohnsituation und Umweltbelastungen sowie die Beschäftigungsverhältnisse und Arbeitsbedingungen zu sehen. Zu berücksichtigen sind weiterhin statusspezifische Unterschiede hinsichtlich des Gesundheitsverhaltens und assoziierter Risiken wie Rauchen, Bewegungsmangel und Übergewicht, der sozialen und personalen Unterstützungs- bzw. Bewältigungsressourcen sowie der Inanspruchnahme der gesundheitsbezogenen Versorgung, einschließlich von Angeboten der Prävention und Gesundheitsförderung (Mielck 2005, Richter, Hurrelmann 2009, Lampert et al. 2016). Die stärkeren Belastungen und das höhere Erkrankungsrisiko in der niedrigen Statusgruppe korrespondieren letztlich mit einer höheren vorzeitigen Sterblichkeit und einer geringeren Lebenserwartung, wobei die Differenz zu den höheren Statusgruppen in Bezug auf die mittlere Lebenserwartung bei Geburt in Deutschland etwa 5 bis 10 Jahre beträgt (Lampert et al. 2007, Lampert, Kroll 2014).

Die Forschungsbemühungen konzentrierten sich zunächst vor allem auf die Bevölkerung im Erwerbsalter, weil die Arbeitswelt als der zentrale Entstehungsort gesundheitlicher Ungleichheit erachtet wurde. In den letzten Jahren ist zudem ein gestiegenes Interesse am Kindes- und Jugendalter festzustellen, da vorliegende Studien deutlich machen, dass die Weichen für ein langes und gesundes Leben früh gestellt werden und die Lebensverhältnisse, in denen man aufwächst, dabei eine wichtige Rolle spielen (Dragano et al. 2010, Lampert 2010, Lampert et al. 2015). Dem höheren Lebensalter wurde demgegenüber lange Zeit nur geringes Interesse zuteil (Lampert 2009, von dem Knesebeck, Schäfer 2009, von dem Knesebeck et al. 2015). Erst in den letzten Jahren, in dem Maße wie lebensphasen- und lebensverlaufsbe- 
zogene Betrachtungen der gesundheitlichen Ungleichheit an Bedeutung gewonnen haben, ist eine stärkere Forschungstätigkeit zu beobachten. Dies gilt vor allem für die Vereinigten Staaten, Großbritannien und die skandinavischen Länder, in denen die Sozialepidemiologie schon länger etabliert ist (Huisman et al. 2013, Shaw et al. 2014, Fors, Thorslund 2015, Read et al. 2016). Aber auch in Deutschland liegen mittlerweile mehrere Studien vor, die sich mit der gesundheitlichen Ungleichheit im höheren Lebensalter beschäftigt haben (von dem Knesebeck et al. 2003, von dem Knesebeck, Vonneilich 2009, Lampert 2009, Schöllgen et al. 2010, Kümpers 2012, Kruse, Schmitt 2016).

Die bisherigen Forschungsergebnisse deuten darauf hin, dass auch nach dem Alter von 65 Jahren soziale Unterschiede in der Gesundheit und der ferneren Lebenserwartung bestehen. Dies scheint zumindest für die ersten Jahre nach dem Ausscheiden aus dem Erwerbsleben zu gelten. Studien, die auch das weiter fortgeschrittene Lebensalter betrachten, also etwa die Jahre ab dem 8o. Lebensjahr, kommen hingegen zu widersprüchlichen Ergebnissen. Während einige Untersuchungen berichten, dass die sozialen Unterschiede in der Gesundheit fortbestehen, zeigen andere, dass sie deutlich schwächer ausfallen bzw. nicht mehr festzustellen sind (von dem Knesebeck et al. 2015).

Vor diesem Hintergrund widmet sich die vorliegende GBE kompakt-Ausgabe der Frage, ob in Bezug auf die gesundheitliche Situation von älteren Menschen soziale Unterschiede bestehen und wie stark diese ausgeprägt sind. Dazu wird zunächst der internationale Forschungsstand zur gesundheitlichen Ungleichheit im höheren Lebensalter zusammengefasst, um dann die Ergebnisse der bislang in Deutschland zu dieser Thematik durchgeführten Untersuchungen vorzustellen.

In Ergänzung werden neue Befunde der Studie »Gesundheit in Deutschland aktuell« (GEDA) präsentiert. Die Analyse der GEDA-Daten richtete sich insbesondere an der Frage aus, wie sich das Ausmaß der gesundheitlichen Ungleichheit im Altersgang verändert, wobei die Betrachtung bereits im Alter von 45 Jahren ansetzt, um auch Aussagen über Veränderungen beim Übergang vom mittleren ins höhere Lebensalter treffen zu können. Abschließend werden die Ergebnisse der GEDA-Studie in den bisherigen Forschungsstand eingeordnet und diskutiert. Die Diskussion stellt einerseits einen Bezug zum demografischen Wandel her, insbesondere dem Umstand, dass immer mehr Menschen, und zwar auch in den sozial benachteiligten Bevölkerungsgruppen, das höhere Lebensalter erreichen. Andererseits wird auf die vorliegenden Erkenntnisse der Alter(n)sforschung hinsichtlich der Kriterien des gesunden Alterns (»healthy aging«) eingegangen.

\section{Internationaler Forschungsstand}

Die vorliegenden Studien zur gesundheitlichen Ungleichheit im höheren Lebensalter betrachten sowohl die Mortalität und fernere Lebenserwartung als auch das Krankheitsgeschehen und die subjektive Gesundheit. Einen Überblick über Studien zu sozialen Unterschieden in der Mortalität älterer Menschen liefert ein systematisches Review von Huisman und Kollegen, in das insgesamt 44 Studien aus Ländern, die zur WHO Region Europa gehören, einbezogen wurden (Huisman et al. 2013). Die Studien zeigen in weitgehender Übereinstimmung, dass Personen mit niedrigem Sozialstatus auch im fortgeschrittenen Lebensalter einem höheren Mortalitätsrisiko unterliegen als Personen mit mittlerem und vor allem mit hohem Sozialstatus.

Dies lässt sich sowohl für das allgemeine Mortalitätsrisiko als auch für das todesursachenspezifische Mortalitätsrisiko feststellen. Die einbezogenen Studien stammen größtenteils aus Großbritannien und Skandinavien. Aber auch für andere Länder werden soziale Unterschiede in der Mortalität und ferneren Lebenserwartung älterer Menschen berichtet. Ein Beispiel hierfür ist eine Studie aus dem deutschsprachigen Teil der Schweiz. Auf der Basis von Daten der Volkszählung und der Todesursachenstatistik konnte diese zeigen, dass Männer, die ein Studium absolviert haben, im Vergleich zu Männern mit einfachem oder ohne Schulabschluss im Alter von 65 Jahren eine um 3,5 Jahre höhere Lebenserwartung haben. Bei Frauen betrug der entsprechende Unterschied zwischen den Bildungsgruppen 2,7 Jahre (Spoerri et al. 2006).

Interessante Einblicke eröffnen darüber hinaus Studien aus den Vereinigten Staaten. Beispielsweise wurden im Rahmen der »Health and Retirement Study« soziale Unterschiede in der Mortalität und im Auftreten funktioneller Einschränkungen über einen Zeitraum von zehn Jahren untersucht (Shaw et al. 2014). Dabei zeigte sich, dass Personen mit geringem Wohlstand ein deutlich erhöhtes Risiko hatten, im Beobachtungszeitraum zu versterben bzw. eine funktionelle Einschränkung zu erleiden. Die Unterschiede zwischen den Wohlstandsgruppen waren dabei in der letzten Phase des Erwerbslebens (51-65 Jahre) stärker ausgeprägt als nach dem Übergang in den Ruhestand (66+ Jahre). Darüber hinaus sprechen die Befunde der Studie dafür, dass gesundheitsrelevante Verhaltensweisen und verhaltensbezogene Risikofaktoren wie Rauchen, Alkoholkonsum, körperliche Inaktivität und Adipositas einen Beitrag zur Erklärung der sozialen Unterschiede im Mortalitätsgeschehen und im Auftreten von funktionellen Einschränkungen im höheren Lebensalter leisten, wobei der Erklärungsbeitrag je nach Alter, Geschlecht und betrachtetem Gesundheitsoutcome zwischen $5 \%$ und $45 \%$ schwankte (Shaw et al. 2014).

Studien, die das Sterblichkeitsgeschehen mit besonderem Fokus auf die Gruppe der Hochaltrigen untersucht haben, kommen zu uneinheitlichen Ergebnissen (Guilley et al. 2010, Huisman et al. 2013). Während einige Studien darauf hindeuten, dass auch im Alter von 80 und mehr Jahren Personen mit niedrigerer Bildung oder geringerem 
Wohlstand ein erhöhtes Mortalitätsrisiko aufweisen, können andere Studien keine sozialen Unterschiede feststellen. Bei spezifischen Todesursachen, wie z. B. Lungenkrebs, spiegeln sich im Sterblichkeitsgeschehen zudem kohorten- und auch geschlechtsspezifische Unterschiede im Gesundheitsverhalten wider (Mackenbach et al. 2004, Van der Heyden et al. 2009). So haben 80- bis 89-jährige Männer mit niedriger Bildung in Ländern wie England/Wales, Belgien und Österreich ein höheres Mortalitätsrisiko für Lungenkrebs als Gleichaltrige mit hoher Bildung, während bei Frauen in denselben Ländern zumindest der Tendenz nach ein umgekehrter Zusammenhang besteht (Mackenbach et al. 2004).

Auch in der subjektiven Gesundheit zeichnen sich im höheren Lebensalter deutliche Unterschiede zuungunsten sozial benachteiligter Bevölkerungsgruppen ab (Read et al. 2016). In einer systematischen Literaturübersicht, in die 71 europäische Studien einflossen, die im Zeitraum von 1995 bis 2013 publiziert wurden, zeigen Read und Kollegen, dass zwischen Indikatoren der sozialen Lage, z. B. Einkommen, Vermögen und der Wohnsituation, und Indikatoren der subjektiven Gesundheit, z. B. allgemeine Lebenszufriedenheit, gesundheitsbezogene Lebensqualität und Selbsteinschätzung des allgemeinen Gesundheitszustandes, zumeist ein enger Zusammenhang besteht: Je prekärer die soziale Lage, desto höher ist auch der Anteil der Älteren, die ihren Gesundheitszustand negativ bewerten und eine geringe Lebensqualität berichten. Dies gilt den Studien zufolge für Frauen und Männer in ähnlicher Weise.

Bezüglich der Veränderung im Altersgang gelangen die Autoren zu der Einschätzung, dass sich die sozialen Unterschiede in der subjektiven Gesundheit mit zunehmendem Alter etwas abschwächen (Read et al. 2016). Einzelne Studien legen allerdings eine andere Schlussfolgerung nahe. So zeigen aktuelle Ergebnisse der Swedish Panel Study of Living Conditions of the Oldest Old (SWEOLD), dass auch in der Gruppe der 77-Jährigen und Älteren deutliche Bildungsunterschiede in der subjektiven Gesundheit bestehen, die sich zudem über den Zeitraum von 1992 bis 2011 als überaus stabil erwiesen haben (Fors, Thorslund 2015).

Hinzuweisen ist darüber hinaus auf die Ergebnisse der europäischen Vergleichsstudie SHARE (»Survey of Health, Ageing and Retirement in Europe«). Diese zeigen unter anderem, dass Personen mit niedriger Bildung im höheren Lebensalter ihren allgemeinen Gesundheitszustand häufiger als weniger gut einschätzen im Vergleich zu Personen mit höherer Bildung (Avendano et al. 2005). Außerdem haben sie ein höheres Risiko für neuauftretende Gesundheitsprobleme und Funktionseinschränkungen im Altersverlauf (Avendano et al. 2009). Besondere Beachtung verdient eine Auswertung der SHARE-Daten aus den Jahren 2004 und 2007, die Längsschnittdaten aus elf Ländern nutzt und der Frage nachgeht, ob sich gesundheitliche Unterschiede zwischen verschiedenen Bildungsgruppen im Alter vergrößern, verringern oder ob sie konstant bleiben. Die Ergebnisse zu zwölf verschiedenen Indikatoren der körperlichen, psychischen und kognitiven Gesundheit legen nahe, dass sich die Unterschiede zwischen den Bildungsgruppen in der betrachteten Altersspanne von 50 bis 80 Jahren in vielen Bereichen ausweiten. Dies trifft $\mathrm{z}$. B. für die Indikatoren Greifkraft, depressive Symptome, numerische Fähigkeiten, zeitliche Orientierung, Mobilität, und Einschränkungen bei instrumentellen Aktivitäten des täglichen Lebens zu. Für einzelne Indikatoren sind jedoch auch kontinuierliche (Anzahl chronischer Krankheiten, subjektive Gesundheitseinschätzung, Gedächtnisleistung) oder konvergierende Verlaufsmuster (Sprechgeschwindigkeit) festzustellen (Leopold, Engelhardt 2011).

\section{Bisherige Befunde für Deutschland}

Der Forschungsstand in Deutschland ist zwar nicht gleichermaßen entwickelt wie z. B. in Großbritannien und Skandinavien, einzelne aussagekräftige Studien liegen aber zwischenzeitlich vor. Beispielsweise belegen Daten des Soziooekonomischen Panels (SOEP) für den Untersuchungszeitraum 1984-2010 deutliche Unterschiede in der Lebenserwartung älterer Menschen in Abhängigkeit vom Einkommen (Kroh et al. 2012). Den Ergebnissen entsprechend beträgt die Differenz in der ferneren Lebenserwartung ab 65 Jahren zwischen der niedrigen und hohen Einkommensgruppe 3,5 Jahre bei Frauen und etwas mehr als 5 Jahre bei Männern. Diese Unterschiede lassen sich der Studie zufolge zumindest teilweise auf eine erhöhte psychische und physische Belastung im Lebenslauf, insbesondere im Erwerbsleben, sowie auf geringere materielle, kulturelle und soziale Ressourcen in der unteren Einkommensgruppe zurückführen.

Soziale Unterschiede in der ferneren Lebenserwartung zuungunsten älterer Menschen mit geringem Einkommen bestanden bereits in der westdeutschen Bevölkerung der 1980er und 1990er Jahre, wie eine Auswertung von Daten des Lebenserwartungssurveys des Bundesinstituts für Bevölkerungsforschung verdeutlicht (Luy et al. 2015). Spätere Daten der Deutschen Rentenversicherung belegen, dass die fernere Lebenserwartung im Alter von ${ }_{5}$ Jahren von Männern in West- und Ostdeutschland mit der Anzahl der in der gesetzlichen Rentenversicherung erworbenen Entgeltpunkte (als Indikator für das Lebenseinkommen) ansteigt (von Gaudecker, Scholz 2007, Shkolnikov et al. 2008, Kibele et al. 2013). Dabei zeigte sich auch, dass sich die Unterschiede im Zeitverlauf vergrößert haben, da Männer mit hohem Lebenseinkommen stärker von einer steigenden Lebenserwartung profitierten als Männer mit sehr niedrigem Einkommen (Kibele et al. 2013).

Darüber hinaus wurde wiederholt gezeigt, dass unter älteren Menschen in Deutschland diejenigen aus sozial benachteiligten Gruppen einen schlechteren subjektiven Gesundheitszustand berichten als jene aus sozial bessergestellten Gruppen (von dem Knesebeck et al. 2003, Lampert 2009, Schöllgen et al. 2010, Wurm et al. 2010). Entsprechende Unterschiede in der Selbsteinschätzung des Gesundheitszustandes lassen sich auch bei chronisch kranken älteren Menschen finden. Dies zeigt eine aktuelle Studie, in die aus- 
schließlich multimorbide Patientinnen und Patienten im Alter von 65 bis 85 Jahren einbezogen wurden. Personen mit höherem Einkommen wiesen deutlich bessere Werte bezüglich ihrer subjektiven Gesundheit auf als Personen mit niedrigerem Einkommen. Auch bei statistischer Kontrolle für den ärztlich eingeschätzten Schweregrad der bestehenden Erkrankungen blieben diese Unterschiede bestehen (von dem Knesebeck et al. 2015).

Für spezifische Krankheiten und Gesundheitsprobleme liegen ebenfalls Befunde vor, die Aufschluss über deren soziale Ungleichverteilung bei Älteren in Deutschland geben. Aus einer Sonderauswertung von Routinedaten der Gmünder Ersatzkasse aus den Jahren 1990 bis 2003 geht hervor, dass 6o- bis 69-jährige pflichtversicherte Frauen und Männer einem höheren Herzinfarktrisiko unterliegen als Gleichaltrige, die freiwillig versichert und damit zumeist ökonomisch besser gestellt sind (Voges et al. 2004). Bei Männern dieser Altersgruppe zeigten sich entsprechende Unterschiede zwischen Pflicht- und freiwillig Versicherten auch hinsichtlich des Lungenkrebsrisikos. Bei Frauen war dies hingegen nicht festzustellen.

Deutschlandweite Ergebnisse der Studie zur Gesundheit Erwachsener in Deutschland aus den Jahren 2008 bis 2011 (DEGS1) machen deutlich, dass Gebrechlichkeit im Alter ebenfalls mit der sozialen Lage zusammenhängt. So weisen 65- bis 79-Jährige mit niedrigem Sozialstatus deutlich häufiger Anzeichen von Gebrechlichkeit auf als Gleichaltrige mit mittlerem und hohem Status (Buttery et al. 2015).

Bezüglich der psychischen Gesundheit zeigen Daten, die in den Jahren 2006 und 2007 im Rahmen des European Social Surveys in Deutschland erhoben wurden, dass depressive Symptome bei Älteren häufiger in bildungsfernen als in bessergebildeten Gruppen vorkommen (von dem Knesebeck, Vonneilich 2009).

Mit Daten der Berliner Altersstudie aus den frühen $1990 e r$ Jahren ließen sich unter anderem soziale Unterschiede in der kognitiven Leistungsfähigkeit älterer Menschen zuungunsten von sozioökonomisch Benachteiligten nachweisen. In der Gruppe der 70- bis 79-jährigen Männer fand sich darüber hinaus ein stark ausgeprägter sozialer Gradient im Vorkommen von Multimorbidität, während bei Frauen diesbezüglich keine sozialen Unterschiede auszumachen waren (Lampert 2000). Zudem ist davon auszugehen, dass Frauen und Männer aus sozial schlechter gestellten im Vergleich zu jenen aus besser gestellten Gruppen einem höheren Risiko unterliegen, pflegebedürftig zu werden, was sowohl aus Krankenkassen- als auch aus Befragungsdaten hervorgeht (Borchert 2008, Unger et al. 2015).

Die Frage, ob und wie sich das Ausmaß gesundheitlicher Ungleichheit im Altersgang verändert, wurde in Deutschland bislang nur selten untersucht (von dem Knesebeck et al. 2003, Schöllgen et al. 2010, Schmidt et al. 2012). Ergebnisse des Deutschen Alterssurveys weisen für bestimmte Gesundheitsbereiche auf eine Ausweitung der sozialen Unterschiede in der Gesundheit über den Altersverlauf hin. So zeigte sich, dass vermögensbezogene Unterschiede im Vorkommen körperlicher Krankheiten und Funktionseinschränkungen im hohen Alter größer ausfielen als im mittleren (Schöllgen et al. 2010). Dies war auch im Hinblick auf Unterschiede in der körperlichen Funktionsfähigkeit zwischen verschiedenen Bildungsgruppen festzustellen (Wurm et al. 2010). Einkommensbezogene Unterschiede in der subjektiven Gesundheit verringerten sich hingegen im Altersgang (Schöllgen et al. 2010). In einer anderen Untersuchung aus Deutschland war ebenfalls eine Verringerung sozialer Unterschiede in der Gesundheit im Altersverlauf zu erkennen, diesmal im Hinblick auf die körperliche und psychische Funktionsfähigkeit (Schmidt et al. 2012).

Zusammenfassend lässt sich somit feststellen, dass die Forschungsergebnisse für Deutschland wie auch der internationale Forschungsstand darauf schließen lassen, dass die gesundheitliche Ungleichheit, die im mittleren Lebensalter einen starken Ausdruck erfährt, auch im höheren Lebensalter beobachtet werden kann. Die vorliegenden Befunde zu der Frage, wie sich die sozialen Unterschieden in der Gesundheit im Verlauf des höheren Alters entwickeln, geben hingegen kein einheitliches Bild wider. Vereinzelt finden sich zwar Hinweise auf eine Verringerung der gesundheitlichen Ungleichheit im fortgeschrittenen Alter. In der Gesamtschau der Studien überwiegt aber der Eindruck, dass das Muster der Veränderung im Altersgang je nach betrachtetem Gesundheitsbereich, den gegenübergestellten Altersgruppen und zum Teil auch nach Geschlecht variiert (von dem Knesebeck, Schäfer 2009).

\section{Aktuelle Ergebnisse der GEDA-Studie}

Für eine aktuelle Betrachtung der gesundheitlichen Ungleichheit in der älteren Bevölkerung Deutschlands wird im Folgenden auf Daten der Studie »Gesundheit in Deutschland aktuell« (GEDA) zurückgegriffen (Lange et al. 2015). Um Informationen von möglichst vielen Personen einbeziehen zu können, wurden für die Analysen Daten aus drei Erhebungswellen zusammengefasst (siehe Infokasten).

Der Gesundheitszustand wurde in allen drei Erhebungswellen unter anderem mit dem Minimum European Health Module (MEHM) erfasst. Dieses aus drei Fragen bestehende Instrument wird auch in europäischen Datenerhebungen wie der Gemeinschaftsstatistik über Einkommen und Lebensbedingungen »Leben in Europa « (EU-SILC) und der Europäischen Gesundheitsumfrage (EHIS) eingesetzt. Die erste Frage des MEHM bezieht sich auf den selbsteingeschätzten allgemeinen Gesundheitszustand, welcher sich in vielen Studien als wichtiger Einflussfaktor der Inanspruchnahme von Gesundheitsleistungen und als starker Prädiktor der Sterblichkeit erwiesen hat (Idler, Benyamini 1997, Miilunpalo et al. 1997, Müters et al. 2005, DeSalvo et al. 2006, Berger et al. 2015). Mit der zweiten Frage wird ermittelt, ob eine oder mehrere chronische Krankheiten, die ständiger Behandlung und Kontrolle bedürfen, vorliegen. Die dritte Frage dient dazu, gesundheitlich bedingte Einschränkungen bei 


\section{Gesundheit in Deutschland aktuell (GEDA) \\ Datenhalter: \\ Ziele: \\ Erhebungsmethode: \\ Grundgesamtheit: \\ Robert Koch-Institut Bereitstellung aktueller Daten zu gesundheitsbezogenen Themen, Analyse zeitlicher Entwicklungen und Trends \\ Computerunterstützte telefonische Befragung (CATI) 18-jährige und ältere Wohnbe- völkerung Deutschlands}

Stichprobe des über die

drei Erhebungswellen

gepoolten Datensatzes:

Responserate:

Untersuchungszeitraum:
34.573 Frauen und 28.033 Männer ab 18 Jahre; 20.140 Frauen und 15.917 Männer ab 45 Jahre; 7.577 Frauen und 5.816 Männer ab 65 Jahre; 2.798 Frauen und 1.768 Männer ab 75 Jahre 29,1 \%, 28,9\% bzw. 22,1\%

GEDA 2009 (Juli 2008 bis Mai 2009), GEDA 2010 (September 2009 bis Juli 2010) und GEDA 2012 (März 2011 bis März 2012) der Verrichtung von Alltagsaktivitäten, die dauerhaft, also seit mindestens einem halben Jahr bestehen, zu erheben. Gesundheitlich bedingte Aktivitätseinschränkungen gelten über den selbsteingeschätzten allgemeinen Gesundheitszustand hinaus als eigenständiger Einflussfaktor der Sterblichkeit (Berger et al. 2015). Zudem sind sie mit erhöhten Ausgaben für gesundheitliche Versorgung verbunden, selbst wenn keine chronische Erkrankung vorliegt (Van der Heyden et al. 2015).

Den zusammengefassten Daten der GEDA-Studien 2009, 2010 und 2012 zufolge schätzen 51,9\% der Frauen im Alter ab 65 Jahren und 46,0 \% der Männer dieser Altersgruppe ihren allgemeinen Gesundheitszustand als mittelmäßig, schlecht oder sehr schlecht ein. Dabei zeigt sich ein ausgeprägter sozialer Gradient zu Ungunsten von Personen mit niedrigerem Sozialstatus. Je niedriger der soziale Status ist, desto höher ist die Prävalenz einer mittelmäßigen bis sehr schlechten Gesundheit von Frauen und Männern im höheren Lebensalter (Abbildung 1). Werden Alterseinflüsse statistisch kontrolliert, ist das Risiko für einen mittelmäßigen bis sehr schlechten allgemeinen Gesundheitszustand bei 65-jährigen und älteren Frauen und Männern mit niedrigem Sozialstatus gegenüber Gleichaltrigen mit hohem Sozialstatus mehr als dreifach erhöht (Frauen: Odds Ratio $[\mathrm{OR}]=3,45$; 95\%-Konfidenzintervall $[\mathrm{KI}]=2,83-4,19$; Männer: $\mathrm{OR}=3,06$; $95 \%-\mathrm{KI}=2,44-3,85)$. Auch bei Personen mit mittlerem Sozialstatus liegt dieses Risiko höher als bei Gleichaltrigen mit hohem Status (Frauen: OR=2,21; 95\%-KI=1,88-2,6o; Männer: OR=1,79; $95 \%-\mathrm{KI}=1,55-2,07)$.

Ähnliche Ergebnisse zeigen sich für die Prävalenz von gesundheitlich bedingten Aktivitätseinschränkungen im
Alltag. Nach eigenen Angaben sind 54,5\% der Frauen und 50,2\% der Männer im Alter ab 65 Jahren aufgrund ihres Gesundheitszustands dauerhaft in der Ausübung ihrer alltäglichen Aktivitäten eingeschränkt. In der niedrigen und in der mittleren Sozialstatusgruppe liegen diese Anteile bei beiden Geschlechtern deutlich höher als in der hohen Statusgruppe (Abbildung 1). Bei statistischer Kontrolle des Alterseinflusses haben 65-jährige und ältere Frauen und Männer mit niedrigem Sozialstatus ein mehr als zweifach erhöhtes Risiko für gesundheitlich bedingte Aktivitätseinschränkungen im Vergleich zu denjenigen mit hohem Sozialstatus (Frauen: $\mathrm{OR}=2,33$; 95\%-KI=1,93-2,81; Männer: $\mathrm{OR}=2,46$; $95 \%-\mathrm{KI}=1,95-3,09)$. Bei Personen mit mittlerem Sozialstatus ist dieses Risiko ebenfalls erhöht (Frauen: $\mathrm{OR}=1,65$; 95\%-KI=1,42-1,92; Männer: OR=1,79; 95\%-KI=1,55-2,05).

Hinsichtlich der Verbreitung von chronischen Krankheiten in der Bevölkerung ab 65 Jahren ist anhand der GEDADaten festzustellen, dass 59,1\% der Frauen und 54,4\% der Männer aus dieser Altersgruppe von mindestens einer chronischen Erkrankung betroffen sind. Dies gilt bei Frauen allerdings nicht für alle Sozialstatusgruppen gleichermaßen. Wie Abbildung 1 verdeutlicht liegt der Anteil chronisch kranker Frauen im Alter ab 65 Jahren in der niedrigen und in der mittleren sozialen Statusgruppe höher als in der hohen Statusgruppe. Werden Altersunterschiede zwischen den Statusgruppen statistisch ausgeglichen, haben 65-jährige oder ältere Frauen mit niedrigem und mittlerem Sozialstatus im Verhältnis zu jenen mit hohem Sozialstatus ein um den Faktor 1,6 (OR=1,61; 95\%-KI=1,34-1,95) bzw. 1,5 $(\mathrm{OR}=1,46 ; 95 \%-\mathrm{KI}=1,26-1,70)$ erhöhtes Risiko für mindestens eine chronische Krankheit. Bei Männern ab 65 Jahren

\section{Messung des sozialen Status}

Der soziale Status wird in den GEDA-Studien und allen anderen Studien des Robert Koch-Instituts einheitlich auf der Basis von Angaben zur schulischen und beruflichen Bildung, zur beruflichen Stellung sowie zum Netto-Äquivalenzeinkommen bestimmt.

Das Netto-Äquivalenzeinkommen wird ausgehend vom Haushaltsnettoeinkommen, also dem Einkommen aller Haushaltsmitglieder abzüglich von Steuern und Sozialabgaben berechnet, wobei die Anzahl der Haushaltsmitglieder und deren, entsprechend ihres Alters geschätzten, Einkommensbedarf berücksichtigt wird (BMAS 2013).

In die Berechnung des Statusindex gehen die drei Sozialmerkmale gleichberechtigt ein. Der Statusindex wird folglich als mehrdimensionaler, ungewichteter Punktsummenscore gebildet, der zwischen 3 und 21 Punkten annehmen kann. Auf dieser Grundlage werden verteilungsbasiert drei Gruppen abgegrenzt, wobei die niedrige Statusgruppe 20\%, die mittlere Statusgruppe 60\% und die hohe Statusgruppe 20\% der Gesamtbevölkerung umfasst (Lampert, Kroll 2009, Lampert et al. 2013b). 
Abbildung 1

Selbsteingeschätzter Gesundheitszustand ("mittelmäßig“, „schlecht“ oder „sehr schlecht“), gesundheitlich bedingte Aktivitätseinschränkungen im Alltag und chronische Erkrankung bei 65-jährigen und älteren Frauen und Männern nach sozialem Status Datenbasis: GEDA 2009, 2010 und 2012
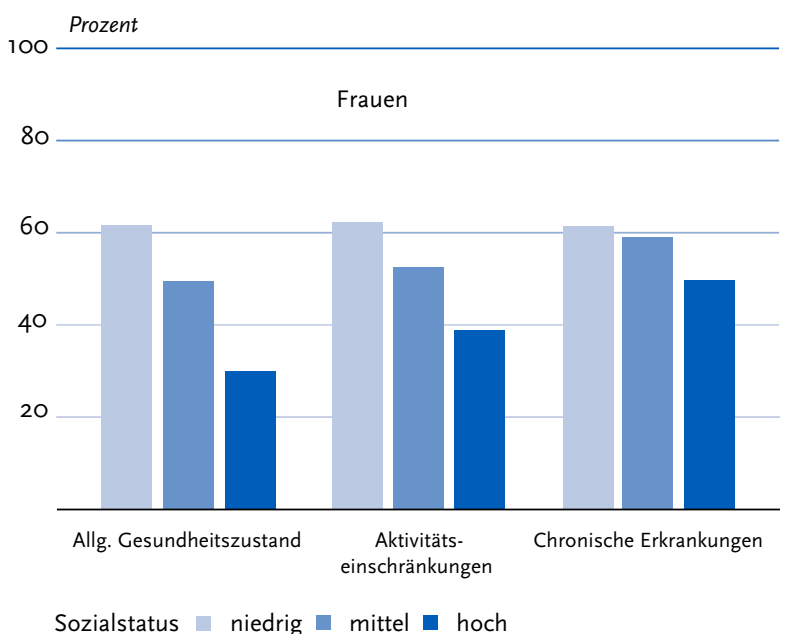

zeichnet sich hingegen nur für jene mit mittlerem Sozialstatus ein 1,2-fach erhöhtes Risiko (OR=1,16; $95 \%$-KI=1,01-1,33) für mindestens eine chronische Krankheit im Vergleich zu Männern mit hohem Sozialstatus ab, wenn für Alterseffekte kontrolliert wird. Für Männer mit niedrigem sozialem Status fällt die Risikoerhöhung gegenüber Gleichaltrigen mit hohem Status hingegen nicht statistisch signifikant aus (OR=1,22; $95 \%-\mathrm{KI}=0,98-1,53$ ).

Um mögliche Veränderungen im Ausmaß gesundheitlicher Ungleichheit beim Übergang vom mittleren ins höhere Alter und dann im weiteren Altersgang zu untersuchen, werden im Folgenden vier Altersgruppen betrachtet: 45 bis 54 Jahre, 55 bis 64 Jahre, 65 bis 74 Jahre und $75+$ Jahre. Bezüglich des selbsteingeschätzten Gesundheitszustandes ist festzustellen, dass bei Frauen die im mittleren Lebensalter stark ausgeprägten Unterschiede
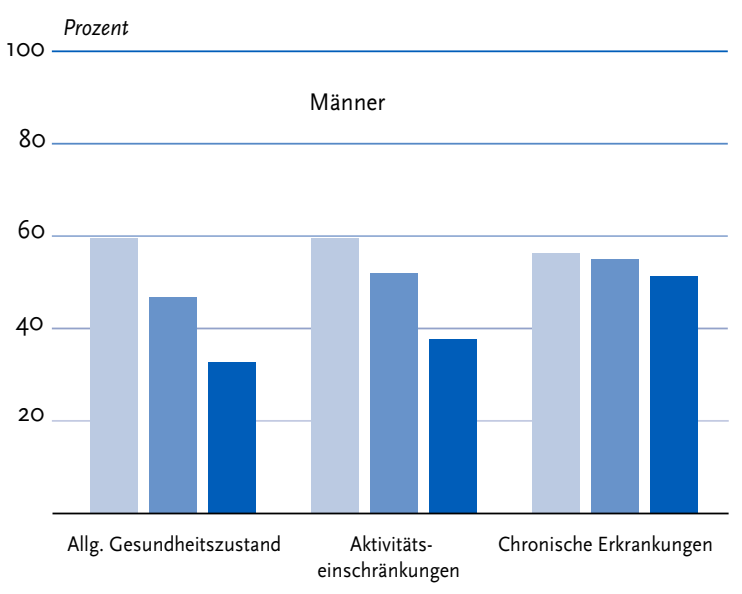

zuungunsten der niedrigen Statusgruppe bis ins hohe Alter fortbestehen. Bei Männern hingegen fallen die statusspezifischen Unterschiede im Alter von 65 bis 74 Jahren etwas und im Alter ab 75 Jahren deutlich schwächer aus als im mittleren Lebensalter (Abbildung 2). Ein ähnliches Muster in der Entwicklung der gesundheitlichen Ungleichheit im Altersgang ist mit Blick auf gesundheitlich bedingte Aktivitätseinschränkungen zu beobachten. Im mittleren Lebensalter zeichnet sich sowohl bei Frauen als auch bei Männern ein deutlicher sozialer Gradient zuungunsten der niedrigen im Vergleich zur mittleren und vor allem zur hohen Statusgruppe ab (Abbildung 3). Bei Frauen erweist sich dieser bis ins hohe Alter als stabil, während bei Männern, insbesondere in der Altersgruppe der 75-Jährigen und Älteren, eine Verringerung der Unterschiede festzustellen ist. Zwischen der niedrigen

\section{Abbildung 2}

Selbsteingeschätzter Gesundheitszustand (»mittelmäßig«, »schlecht« oder »sehr schlecht«) bei Frauen und Männern nach sozialem Status und Altersgruppe

Datenbasis: GEDA 2009, 2010, 2012
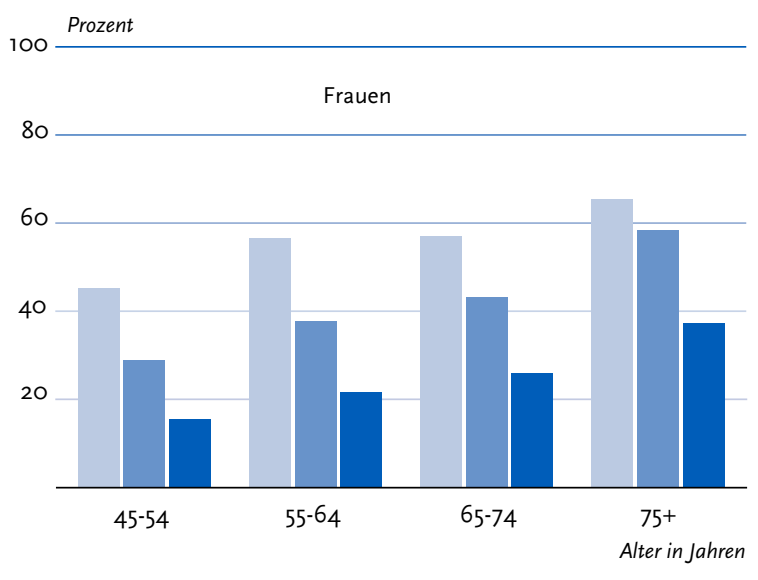

Sozialstatus niedrig mittel $\square$ hoch
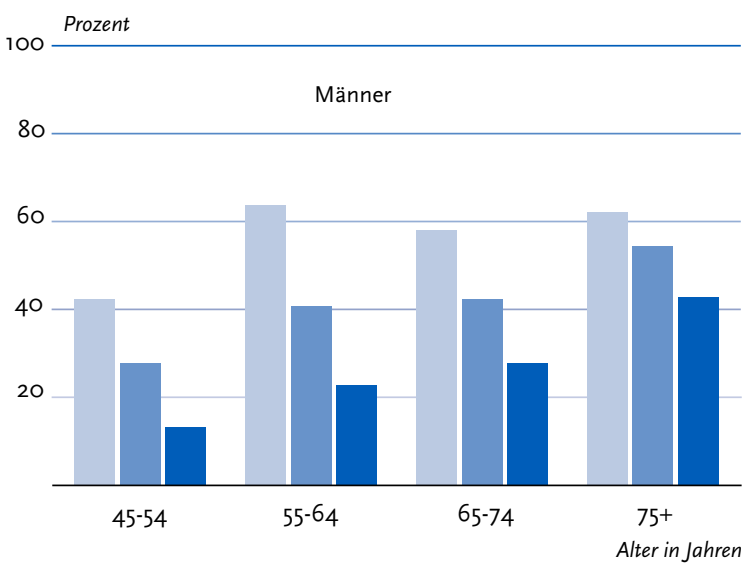
Abbildung 3

Gesundheitlich bedingte Einschränkungen im Alltag bei Frauen und Männern nach sozialem

Status und Altersgruppe

Datenbasis: GEDA 2009, 2010 und 2012

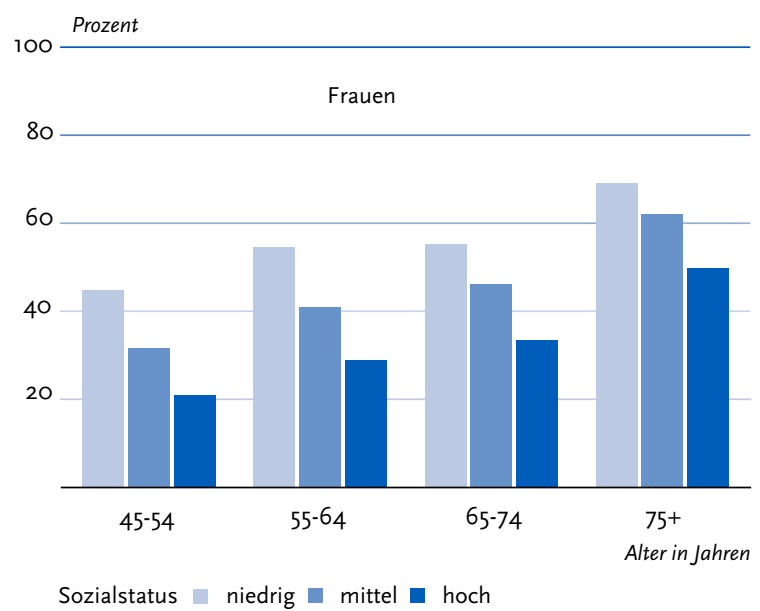

und mittleren Statusgruppe bestehen in dieser Altersgruppe keine Unterschiede mehr. Auch in Bezug auf die Verbreitung chronischer Krankheit treten im mittleren Lebensalter deutliche Unterschiede zwischen den Statusgruppen zutage (Abbildung 4).

Im Gegensatz zum selbsteingeschätzten Gesundheitszustand und gesundheitlich bedingten Einschränkungen in der Alltagsaktivität zeichnet sich in der Verbreitung chronischer Krankheit bei beiden Geschlechtern eine deutliche Verringerung der statusspezifischen Unterschiede im Altersgang ab, die bereits in der Altersgruppe der 65- bis 74-Jährigen auszumachen ist. Ein geschlechtsspezifischer Unterschied ist ab dem Alter von 75 Jahren zu beobachten, da bei Männern anders als bei Frauen keine signifikanten statusspezifischen Unterschiede mehr bestehen.
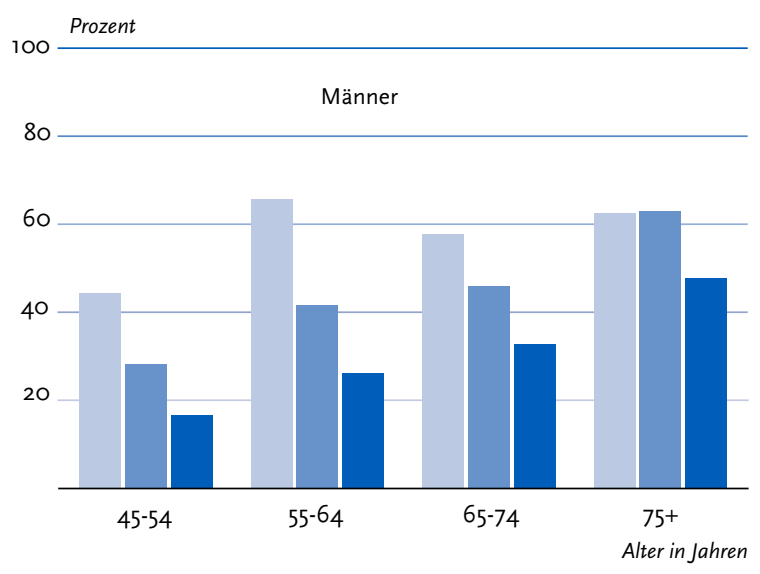

\section{Diskussion}

Die bisherigen Ergebnisse zu sozialen Unterschieden in der Gesundheit und ferneren Lebenserwartung im höheren Lebensalter und deren Veränderung im Altersgang werden in der Literatur mit Bezug auf drei Hypothesen diskutiert, die von einem weitgehend unveränderten Fortbestehen (»Kontinuität«), einer Ausweitung (»Divergenz«) bzw. einer Verringerung oder sogar Angleichung (»Konvergenz«) der gesundheitlichen Ungleichheit ausgehen (Lampert 2009, von dem Knesebeck, Vonneilich 2009, Leopold, Engelhardt 2011). Für die »Kontinuitätsthese« spricht, dass sich der soziale Status beim Übergang vom mittleren ins höhere Lebensalter zumeist nicht ändert. Im jungen und mittleren Lebensalter finden zahlreiche Entwicklungen z. B. in Bezug auf Bildungsweg, Erwerbsverlauf oder Familienleben statt, die mit Veränderungen der sozioökonomischen Situation

Abbildung 4

Chronische Erkrankung bei Frauen und Männern nach sozialem Status und Altersgruppe

Datenbasis: GEDA 2009, 2010, 2012
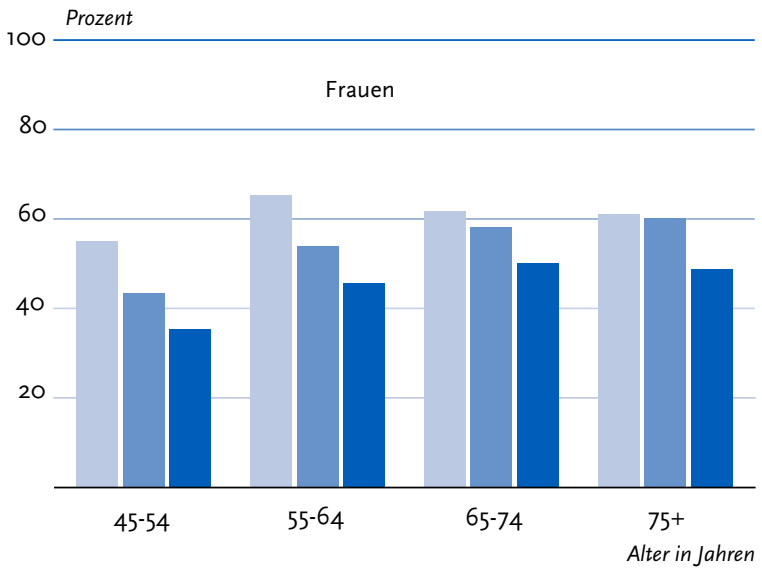

Sozialstatus niedrig $\square$ mittel $\square$ hoch
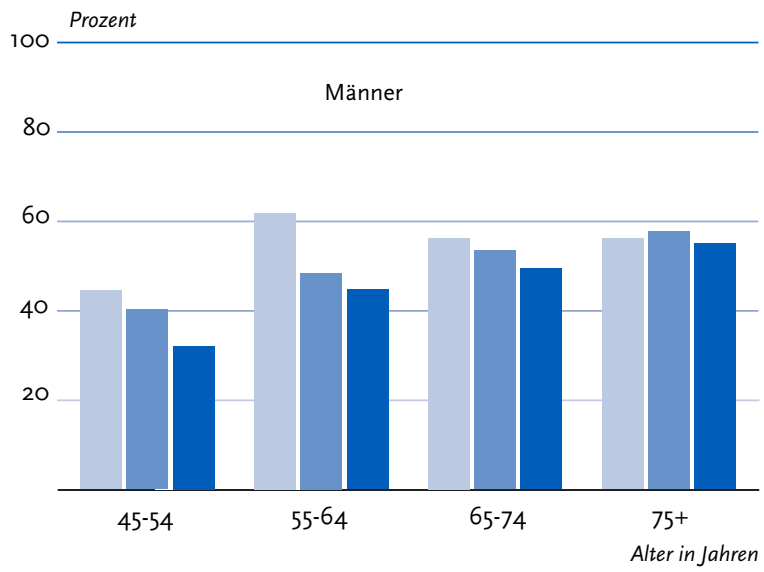
einhergehen. Auch der Übergang in das höhere Lebensalter ist durch Änderungen der Lebenssituation gekennzeichnet, schon allein durch das Ausscheiden aus dem Erwerbsleben. Gemäß der »Kontinuitätsthese« wird aber angenommen, dass sich der soziale Status dabei als relativ stabil erweist, auch aufgrund der gesetzlichen Ruhestandsregelungen. Ebenso wird in Bezug auf viele Prozesse und Mechanismen, die der gesundheitlichen Ungleichheit zugrunde liegen, unterstellt, dass diese bis ins höhere Lebensalter überdauern. Als Beispiele hierfür werden gesundheitsbezogene Einstellungen, Wahrnehmungen und Verhaltensweisen, personale Ressourcen und Bewältigungsstrategien sowie soziale Beziehungen und Unterstützungsleistungen genannt.

Die »Divergenzthese « knüpft an die Argumente der »Kontinuitätsthese« an, zieht aber andere Schlussfolgerungen. In Bezug auf die Lebensbedingungen, das Gesundheitsverhalten sowie personale und soziale Ressourcen wird davon ausgegangen, dass die Stabilität im Altersgang $z u$ einer Ausweitung der gesundheitlichen Ungleichheit führt. Beispielsweise wird angenommen, dass mit dem Alter einhergehende Verluste und Einbußen die Angehörigen der unteren Statusgruppen stärker oder zumindest früher betreffen. Dies dürfte z. B. für den Verlust sozialer Rollen, den Tod nahestehender Personen oder das verstärkte Auftreten von chronischen Krankheiten und Beschwerden gelten. Zudem verfügen Personen mit niedrigem Sozialstatus über geringere ökonomische, soziale und personale Ressourcen, um daraus erwachsende Probleme und Nachteile zu bewältigen oder zu kompensieren.

Auch die gesetzlichen Ruhestandsregelungen tragen nach der »Divergenzthese « eher zu einer Ausweitung der sozialen und der gesundheitlichen Ungleichheit bei, zumal in den höheren Statusgruppen deutlich häufiger zusätzlich auf eine private Altersvorsorge oder finanzielle Rücklagen zurückgegriffen werden kann. Außerdem weisen die Angehörigen der niedrigen Statusgruppen häufiger brüchige Erwerbsbiografien und infolgedessen im fortgeschrittenen Lebensalter ein höheres Armutsrisiko auf. Darüber hinaus geht die »Divergenzthese « von einer Kumulation gesundheitlicher Belastungen und Risiken über den gesamten Lebensverlauf aus. Hinsichtlich verhaltensbezogener Risikofaktoren wie Rauchen, riskanter Alkoholkonsum, Bewegungsmangel und Fehlernährung, die in den niedrigen Statusgruppen deutlich stärker verbreitet sind, ist evident, dass der Einfluss auf die Gesundheit umso größer ist, je länger sie wirksam sind. Gleiches kann für Konflikte und Stressbelastungen, z. B. in der Familie oder im weiteren sozialen Umfeld, die nicht selten ökonomische Gründe haben und aus einer dauerhaften materiellen Notlage resultieren, angenommen werden. Bei der Entstehung vieler der im Alter verstärkt auftretenden körperlichen und psychischen Krankheiten sowie daraus resultierenden Funktionseinschränkungen spielt die Kumulation von Belastungen und Risiken im Lebenslauf eine wichtige Rolle.

Im Gegensatz zur »Divergenzthese« geht die » Konver- genzthese davon aus, dass die gesundheitliche Ungleichheit im höheren Lebensalter abnimmt und es in Bezug auf die körperlichen, psychischen und funktionellen Fähigkeiten zu einer schrittweisen Annäherung zwischen den sozialen Statusgruppen kommt. Die Argumente, die zur Unterstützung der »Kontinuitäts-« und »Divergenzthese« vorgebracht werden, müssen dabei nicht infrage gestellt werden. Vielmehr bezieht sich die »Konvergenzthese « auf zwei Prozesse, von denen angenommen wird, dass sie die Kontinuität des sozialen Status und der Lebensbedingungen bzw. die Kumulation von gesundheitsbezogenen Belastungen und Risiken überlagern. Zum einen wird auf Prozesse des biologischen Alterns verwiesen, in deren Folge sich die organischen Kapazitätsreserven vermindern und die Vulnerabilität und Krankheitsanfälligkeit des menschlichen Organismus zunimmt. Altersspezifische physiologische Veränderungen und pathologische Prozesse können zwar interindividuell unterschiedlich ausgeprägt sein und verlaufen, im sehr hohen Alter - so die Annahme - verringert sich die Variationsbreite aber zusehends. Mit Blick auf das Krankheits- und Sterbegeschehen im hohen Alter wird darauf verwiesen, dass extreme Langlebigkeit bestimmte genetische Dispositionen voraussetzt (Christensen, Vaupel 1996), die unabhängig von sozioökonomischen Merkmalen verteilt sind. Aufgrund dessen wird angenommen, dass soziale Unterschiede in der Gesundheit und ferneren Lebenserwartung von Hochbetagten allenfalls sehr schwach zutage treten.

Zum anderen wird die Verringerung der gesundheitlichen Ungleichheit im hohen Alter mit Prozessen des selektiven Überlebens (»selective survival«) begründet (Markides, Machalek 1984, Wing et al. 1985). Aufgrund einer höheren vorzeitigen Sterblichkeit wird in den niedrigen Statusgruppen im Vergleich zu den mittleren und hohen Statusgruppen ein geringerer Anteil 65 Jahre oder älter. Die vorzeitige Sterblichkeit betrifft vor allem Personen, die schon früh mit Krankheiten und Gesundheitsproblemen zu tun haben. Infolge dessen könnten die Angehörigen der niedrigeren Statusgruppen im höheren Lebensalter in Bezug auf ihre genetische Veranlagung, organische Vulnerabilität, aber auch in Bezug auf ihren Lebensstil und ihre personalen und sozialen Ressourcen eine positiv selektierte Gruppe darstellen. Die statusspezifischen Unterschiede in der Gesundheit und ferneren Gesundheit dürften dann entsprechend geringer ausfallen.

Die vorgestellten Ergebnisse der GEDA-Studie unterstützen die »Kontinuitäts-« und die »Konvergenzthese«, wobei sich Hinweise auf eine Verringerung der gesundheitlichen Ungleichheit eher bei Männern als bei Frauen finden. Ein Grund hierfür könnte sein, dass die vorzeitige Sterblichkeit bei Männern deutlich höher ist als bei Frauen. Eine Verringerung der gesundheitlichen Ungleichheit ist deshalb bei Frauen erst im weiter fortgeschrittenen Lebensalter zu erwarten. Dies kann mit den Daten der GEDA-Studie nicht untersucht werden, weil die höheren Altersjahrgänge trotz der insgesamt großen Stichprobe nur sehr schwach besetzt sind. 
Auch der bisherige nationale und internationale Forschungsstand unterstützt die »Kontinuitäts-« und »Konvergenzthese $«$. Viele Studien, die eine altersdifferenzierte Betrachtung angestellt haben, sprechen dafür, dass zumindest in den ersten Jahren nach dem Übergang in den Ruhestand die sozialen Unterschiede in der Gesundheit und ferneren Lebenserwartung stabil bleiben, es dann aber mit fortschreitendem Alter zu einer Verringerung der Unterschiede kommt. Für eine Ausweitung der gesundheitlichen Ungleichheit, wie sie durch die »Divergenzthese« nahegelegt wird, finden sich in den vorgestellten GEDAErgebnissen keine Anhaltspunkte, obwohl die für die These vorgebrachten Argumente, die z. B. auf einen angenommenen Bedeutungsgewinn sozioökonomischer, sozialer und personaler Ressourcen im Alter zielen, möglicherweise insbesondere im Fall von Krankheit und Hilfsbedürftigkeit, durchaus plausibel erscheinen. In anderen Studien fanden sich vereinzelt Hinweise auf eine Ausweitung der gesundheitlichen Ungleichheit im Altersgang, allerdings nur für bestimmte Gesundheitsbereiche und auch eher am Übergang vom mittleren ins höhere Lebensalter und nicht so sehr mit Blick auf die Hochaltrigen (Schöllgen et al. 2010, Leopold, Engelhardt 2011).

Die Ergebnisse zur gesundheitlichen Ungleichheit im höheren Lebensalter sind als Momentaufnahme vor dem Hintergrund der aktuellen demografischen Situation zu sehen. Wie eingangs bereits angesprochen, stellt sich somit die Frage, wie sich das Ausmaß und Erscheinungsbild der gesundheitlichen Ungleichheit in den nächsten Jahrzehnten im Zuge des demografischen Wandels, insbesondere der zunehmenden Alterung der Bevölkerung, verändern wird. Die demografische Alterung wird vor allem an der Veränderung des Durchschnittsalters und dem Anteil älterer Menschen an der Gesamtbevölkerung festgemacht (Menning et al. 2010). Das Durchschnittsalter der Bevölkerung Deutschlands ist seit der Wiedervereinigung um 5,0 Jahre auf 44,3 Jahre im Jahr 2014 angestiegen. Der Anteil der Bevölkerung im Alter von 65 Jahren und älter erhöhte sich im gleichen Zeitraum von 14,9\% auf 21,0\%.

Die demografische Alterung wird von mehreren Faktoren verursacht (Schwarz 1997, Dinkel 2008), von denen hinsichtlich der gesundheitlichen Ungleichheit im höheren Lebensalter insbesondere der nachhaltige Anstieg der Lebenserwartung und die wellenartige Altersstruktur bedeutsam sind. Die Lebenserwartung Neugeborener ist seit Beginn der $1960 e r$ Jahre um etwa zwei Jahre pro Dekade, die fernere Lebenserwartung ab 65 Jahre um etwa ein Jahr pro Dekade angestiegen. Dabei resultiert der Anstieg der Lebenserwartung in den letzten Jahrzehnten insbesondere aus einem Rückgang der Sterblichkeit in den hohen und höchsten Altersgruppen (Doblhammer, Kreft 2011). Für die Zukunft wird erwartet, dass sich dieser Trend eines Anstiegs der Lebenserwartung mit den größten Zugewinnen in den hohen Altersgruppen weiter fortsetzt (Oeppen, Vaupel 2002, Vaupel 2010).

Die heute zu beobachtende wellenartige Altersstruktur ist auf mehrere einschneidende gesellschaftliche Ereignis- se und Prozesse im Laufe des 20. Jahrhunderts zurückzuführen (Bundesinstitut für Bevölkerungsforschung, Statistisches Bundesamt 2008). Im Zusammenhang mit dem Zweiten Weltkrieg sind neben der hohen Zahl vor allem junger männlicher Kriegsopfer auch die Einbrüche in der Geburtenrate von Bedeutung. Nach 1945 stieg die Geburtenrate wieder an und lag in den Jahren 1959 bis 1968 am höchsten. Diese als Babyboomer bezeichnete Generation, die aktuell zwischen 47 und 56 Jahren alt ist, bildet gegenwärtig die am stärksten besetzte Altersgruppe (Menning, Hoffmann 2009). Die so entstandenen »Alterswellen« werden den Prozess der demografischen Alterung noch auf lange Sicht prägen (Scharein 2012).

Die demografische Alterung wird in den nächsten Jahrzehnten weiter anhalten. Vom Jahr 2025 an werden jährlich 1,2 Millionen bis über 1,3 Millionen Menschen die Altersschwelle von $6_{5}$ Jahren überschreiten (13. Koordinierte Bevölkerungsvorausberechnung, Variante G1-L1-W2; Statistisches Bundesamt 2015). Infolge dessen wird die Zahl älterer Menschen deutlich zunehmen, während die Zahl der Menschen in den jüngeren und mittleren Altersgruppen zurückgehen wird. Besonders stark anwachsen wird die Gruppe der Hochaltrigen. Bis 2030 wird die Zahl derjenigen, die 80 Jahre oder älter sind, um $42 \%$ von 4,5 Millionen im Jahr 2014 (3 Millionen Frauen, 1,6 Millionen Männer) auf 6,5 Millionen Menschen im Jahr 2030 (3,9 Millionen Frauen, 2,5 Millionen Männer) zunehmen. Damit sind die Hochaltrigen die am stärksten wachsende Altersgruppe, wobei der Zuwachs bei Männern mit $+60 \%$ noch stärker ausfallen wird als bei Frauen mit $+33 \%$.

Die genannten demografischen Entwicklungen werden alle sozialen Statusgruppen betreffen. Aufgrund dessen werden in Zukunft auch mehr Angehörige sozial benachteiligter Bevölkerungsgruppen das hohe Alter als Lebensphase erreichen. Sollte die »Konvergenzthese« zutreffen und die Entwicklung der gesundheitlichen Ungleichheit im Alter unter anderem das Ergebnis von selektivem Überleben sein, dann ist der Entwicklung der vorzeitigen Sterblichkeit besondere Beachtung zu schenken. Die vorzeitige Sterblichkeit ist seit Jahrzehnten rückläufig, und zwar bei Männern stärker als bei Frauen. Aller Voraussicht nach wird dieser Prozess anhalten, sodass immer mehr Personen der niedrigen Statusgruppen das höhere Lebensalter erreichen.

Ein Teil des Rückgangs der vorzeitigen Sterblichkeit liegt darin begründet, dass immer häufiger potenziell lebensbedrohliche Krankheiten, darunter Herzinfarkt oder Diabetes mellitus, überlebt werden. Diese »Überlebenden« leiden dann aber häufig an Folgekrankheiten und Funktionseinschränkungen, die oftmals mit einem erheblichen Versorgungs- und Hilfebedarf einhergehen. Infolgedessen könnte die im mittleren Lebensalter zu beobachtende gesundheitliche Ungleichheit zunehmend bis ins höhere Lebensalter überdauern und dort in altersspezifischen Krankheiten und Behinderungen zum Ausdruck kommen, und zwar nicht nur bei Frauen, sondern 
zusehends auch bei Männern. Die beschriebenen Auswirkungen des selektiven Überlebens und des biologischen Alterns werden damit nicht außer Kraft gesetzt, könnten aber erst im weiter fortgeschrittenen Alter zu einer Verringerung der gesundheitlichen Ungleichheit führen.

Aus der vorliegenden Bestandsaufnahme zur gesundheitlichen Ungleichheit im höheren Lebensalter und im Lebensverlauf ergeben sich wichtige Implikationen für die Public Health Praxis und Forschung. Zum einen wird deutlich, dass Public Health Maßnahmen zur Stärkung und Sicherung der Gesundheit in der Bevölkerung auf die besonderen Belange sozial benachteiligter Menschen in allen Altersgruppen ausgerichtet sein müssen. In einem kürzlich veröffentlichten Bericht zu Alter und Gesundheit spricht die Weltgesundheitsorganisation (WHO) von der Notwendigkeit eines Public Health-Rahmenkonzeptes für »Healthy Ageing « und von der gesamtgesellschaftlichen Aufgabe, in den Erhalt von Autonomie, Funktionsfähigkeit und Teilhabe von Menschen bis ins hohe Alter hinein $\mathrm{zu}$ investieren (»Investment in and return on investment in ageing populations «) (Word Health Organization 2015). Im Bericht wird ausführlich darauf eingegangen, dass diese Aufgabe weit gefächert ist und nicht nur Gesundheitsförderung, Prävention und gesundheitliche Versorgung im engeren Sinne umfasst. Große Bedeutung wird der sozialen Sicherheit und einer altersgerechten Gestaltung von Lebens-, Wohn- und Arbeitswelten unter politischer Federführung und Koordination beigemessen, ebenso wie der Verbesserung von Bildungsangeboten, der Stärkung von Gesundheitskompetenz (»Health Literacy«) und Selbstwirksamkeit (»Empowerment«).

Einige, wenn auch nicht alle dieser Aspekte werden im nationalen Gesundheitsziel für Deutschland »Gesund älter werden« angesprochen (Kooperationsverbund gesundheitsziele.de 2012). Im Mittelpunkt steht dabei die Zielgruppe der älteren Menschen ab 65 Jahren. $\mathrm{Zu}$ insgesamt drei Handlungsfeldern (I. Gesundheitsförderung und Prävention: Autonomie erhalten, II. Medizinische, psychosoziale und pflegerische Versorgung (Thelen et al. 2012), III. Besondere Herausforderungen) wurden Ziele definiert und Maßnahmen zur Zielerreichung vorgeschlagen. Beispiele sind Maßnahmen zur Stärkung der psychischen Gesundheit und gesellschaftlichen Teilhabe älterer Menschen, Maßnahmen zur Förderung von körperlicher Aktivität und gesunder Ernährung sowie Maßnahmen zur Verbesserung von Versorgungsangeboten und der Versorgungsqualität bei Pflegebedürftigkeit, Demenz und Multimorbidität. Durchgängig wurde bei der Erarbeitung dieses Ziele- und Maßnahmenkataloges die Anforderung gestellt, geschlechtsspezifische Unterschiede und bestimmte Bevölkerungsgruppen, wie z. B. sozial benachteiligte ältere Menschen und ältere Menschen mit Migrationshintergrund in besonderer Weise zu berücksichtigen.

Darüber hinaus zeigen die hier zusammengestellten Ergebnisse, dass nach wie vor großer Forschungsbedarf besteht, um die Auswirkungen der sozialen Ungleichheit auf die Gesundheit der älteren Bevölkerung zu verstehen und wissenschaftliche Evidenz für geeignete und vorausschauende Public Health Strategien zu schaffen. So sind widersprüchliche oder auch lückenhafte Forschungsergebnisse zur gesundheitlichen Ungleichheit im höheren Lebensalter nicht zuletzt darauf zurückzuführen, dass unterschiedliche gesundheitliche Zielgrößen und Facetten der sozialen Lage betrachtet werden und Daten zu hochaltrigen Menschen schlichtweg fehlen. Für die in dieser GBE kompakt-Ausgabe vorgenommene Analyse wurden Daten aus drei Erhebungswellen der GEDA-Studie zusammengespielt, sodass Informationen von mehr als 13.000 Personen im Alter ab 65 Jahren und von über 36.000 Personen im Alter ab 45 Jahren für die Analysen herangezogen werden konnten. $\mathrm{Zu}$ beachten ist jedoch, dass besonders Personen aus den hohen Altersgruppen eine Teilnahme an den telefonisch durchgeführten GEDA-Studien verweigert haben dürften, wie es in Bevölkerungsbefragungen häufig $\mathrm{zu}$ beobachten ist.

Für die Nichtteilnahme an Gesundheitssurveys von älteren Menschen spielen gesundheitliche Gründe eine nicht unerhebliche Rolle (Gaertner et al. 2016). Als Folge kann es zu einer Unterschätzung der Prävalenz von chronischen Erkrankungen und Funktionseinschränkungen kommen, was bei der Interpretation der Ergebnisse berücksichtigt werden sollte. In den dargestellten Analysen wurde allerdings ein Gewichtungsverfahren angewendet, um mögliche Auswirkungen systematischer Nichtteilnahmen auf die Studienergebnisse zu minimieren. Mithilfe des Verfahrens wurde die in allen drei GEDA-Wellen realisierte Stichprobe hinsichtlich Alter, Geschlecht, Bildung und Bundesland an die Verteilung in der Gesamtbevölkerung Deutschlands angepasst. Um in hohen Altersgruppen die Teilnahme an Studien zu verbessern, sind Studienansätze notwendig, die spezifisch auf diese Bevölkerungsgruppe ausgerichtet sind (siehe z. B. Lindenberger et al. 2010). Aufgrund des hohen Aufwandes, der mit solchen Ansätzen verbunden ist, werden derartige Altersstudien bislang zumeist regional begrenzt durchgeführt. Im bereits zitierten WHO-Bericht wird aber hervorgehoben, dass ein indikatorengestütztes kontinuierliches Gesundheitsmonitoring auf Bevölkerungsebene unter Einschluss aller Altersgruppen, einschließlich hochaltriger und gesundheitlich bereits eingeschränkter Menschen, wesentlich dazu beitragen kann, die Evidenzgrundlage zu verbessern (World Health Organization 2015). Der Wert einer solchen Datengrundlage liegt darin, dass umfassende und standardisierte Informationen zu Gesundheitsstatus, zu Gesundheitsrisiken und zur sozialen Lage regelmäßig wiederkehrend erhoben werden. Dies ermöglicht sowohl altersgruppen- oder lebensphasenspezifische Querschnittsanalysen als auch Trendanalysen zu zeitlichen Veränderungen innerhalb bestimmter Untergruppen, z. B. Altersgruppen oder sozialer Statusgruppen.

So ließe sich zusammenfassend beschreiben, ob gesundheitliche Ungleichheit in der Bevölkerung im Hinblick auf Krankheitshäufigkeiten und Multimorbidi- 
tät, funktionelle gesundheitliche Einschränkungen oder bestimmte gesundheitliche Risiken und Ressourcen über die Zeit zu- oder abnimmt bzw. persistiert. Hiermit wäre ein wesentlicher Baustein für die epidemiologische Begleitforschung von Gesundheitszielen und gesundheitspolitischen Maßnahmen gegeben.

PD Dr. Thomas Lampert, Jens Hoebel, Dr. Benjamin Kuntz, Dr. Judith Fuchs, Dr. Christa Scheidt-Nave, Enno Nowossadeck Robert Koch-Institut Abteilung für Epidemiologie und Gesundheitsmonitoring

\section{Literatur}

Avendano M, Aro AR, Mackenbach J (2005) Socio-economic disparities in physical health in 10 European countries. In: Börsch-Supan A, Brugiavini A, Juerges $\mathrm{H}$ et al. (Hrsg) Health, ageing and retirement in Europe. First results from the Survey of Health, Ageing and Retirement in Europe. Mannheim Research Institute for the Economics of Aging, Mannheim, S 89-94

Avendano M, Jürges H, Mackenbach JP (2009) Educational level and changes in health across Europe: longitudinal results from SHARE. J Euro Social Policy 19 (4): 301-316

Berger N, Van der Heyden J, Van Oyen H (2015) The global activity limitation indicator and self-rated health: two complementary predictors of mortality. Arch Public Health 73 (1): 25

Borchert L (2008) Soziale Ungleichheit und Gesundheitsrisiken älterer Menschen: Eine empirische Längsschnittanalyse unter Berücksichtigung von Morbidität, Pflegebedürftigkeit und Mortalität. MaroVerlag, Augsburg

Bundesinstitut für Bevölkerungsforschung, Statistisches Bundesamt (Hrsg) (2008) Bevölkerung. Daten, Fakten, Trends zum demographischen Wandel in Deutschland. BiB, Destatis, Wiesbaden

Buttery AK, Busch MA, Gaertner B et al. (2015) Prevalence and correlates of frailty among older adults: findings from the German health interview and examination survey. BMC geriatrics 15: 22

Christensen K, Vaupel JW (1996) Determinants of longevity: genetic, environmental and medical factors. J Intern Med 240 (6): 333-341

DeSalvo KB, Bloser N, Reynolds K et al. (2006) Mortality prediction with a single general self-rated health question. J Gen Intern Med 21 (3): $267-275$

Dinkel RH (2008) Was ist demographische Alterung? Der Beitrag der demographischen Parameter zur demographischen Alterung in den alten Bundesländern seit 1950. In: Häfner H, Staudinger UM (Hrsg) Was ist Alter(n)? Neue Antworten auf eine scheinbar einfache Frage. Springer, Berlin, S 97-117

Doblhammer G, Kreft D (2011) Länger leben, länger leiden? Bundesgesundheitsblatt - Gesundheitsforschung - Gesundheitsschutz 54 (8): $907-914$

Dragano N, Lampert T, Siegrist J (2010) Wie baut sich soziale und gesundheitliche Ungleichheit im Lebenslauf auf? In: Sachverständigenkommission 13. Kinder- und Jugendbericht (Hrsg) Materialien zum 13. Kinder- und Jugendbericht - Mehr Chancen für gesundes Aufwachsen. Verlag Deutsches Jugendinstitut, München, S 11-50

European Commission (Hrsg) (2013) Health inequalities in the EU - Final report of a consortium. Consortium lead: Sir Michael Marmot. European Commission Directorate-General for Health and Consumers, Brussels

Fors S, Thorslund M (2015) Enduring inequality: educational disparities in health among the oldest old in Sweden 1992-2011. Int J Public Health 60 (1): 91-98

Gaertner B, Seitz I, Fuchs J et al. (2016) Baseline participation in a health examination survey of the population 65 years and older: who is missed and why? BMC geriatrics 16: 21

Galobardes B, Shaw M, Lawlor DA et al. (2006) Indicators of socioeconomic position. In: Oakes JM, Kaufman JS (Hrsg) Methods in social epidemiology. Jossey-Bass, San Francisco, S 47-85

Guilley E, Bopp M, Faeh D et al. (2010) Socioeconomic gradients in mortality in the oldest old: a review. Arch Gerontol Geriatr 51 (3): e37-40 
Hradil S (2001) Soziale Ungleichheit in Deutschland. 8. Auflage. Leske + Budrich, Opladen

Huisman M, Read S, Towriss CA et al. (2013) Socioeconomic inequalities in mortality rates in old age in the World Health Organization Europe region. Epidemiologic Reviews 35 (1): 84-97

Idler EL, Benyamini Y (1997) Self-rated health and mortality: a review of twenty-seven community studies. J Health Soc Behav 38 (1): 21-37

Kibele EUB, Jasilionis D, Shkolnikov VM (2013) Widening socioeconomic differences in mortality among men aged 65 years and older in Germany. J Epidemiol Community Health 67 (5): 453-457

Kooperationsverbund gesundheitsziele.de (Hrsg) (2012) Nationales Gesundheitsziel »Gesund älter werden«. BMG, Berlin

Kroh M, Neiss H, Kroll Let al. (2012) Menschen mit hohen Einkommen leben länger. DIW Wochenbericht 38

https://www.diw.de (Stand: 05.03.2016)

Kruse A, Schmitt E (2016) Soziale Ungleichheit, Gesundheit und Pflege im höheren Lebensalter. Bundesgesundheitsblatt - Gesundheitsforschung - Gesundheitsschutz 59 (2): 252-258

Kümpers S (2012) Sozial bedingte gesundheitliche Ungleichheit im Alter. Public Health Forum 20 (1): 4.e1-4.e3

Lampert T (2000) Sozioökonomische Ungleichheit und Gesundheit im höheren Lebensalter. Alters- und geschlechtsspezifische Differenzen. In: Backes GM, Clemens W (Hrsg) Lebenslagen im Alter: Gesellschaftliche Bedingungen und Grenzen. Leske + Budrich, Opladen, S 159-181

Lampert T (2009) Soziale Ungleichheit und Gesundheit im höheren Lebensalter. In: Böhm K, Tesch-Römer C, Ziese T (Hrsg) Gesundheit und Krankheit im Alter. Eine gemeinsame Veröffentlichung des Statistischen Bundesamtes, des Deutschen Zentrums für Altersfragen und des Robert Koch-Instituts. RKI, Berlin, S 121-133

Lampert T (2010) Frühe Weichenstellung. Zur Bedeutung der Kindheit und Jugend für die Gesundheit im späteren Leben. Bundesgesundheitsblatt Gesundheitsforschung Gesundheitsschutz 53 (5): 486-497

Lampert T, Kroll LE (2009) Die Messung des sozioökonomischen Status in sozialepidemiologischen Studien. In: Richter M, Hurrelmann K (Hrsg) Gesundheitliche Ungleichheit Grundlagen, Probleme, Perspektiven. 2, aktualisierte Auflage. VS Verlag für Sozialwissenschaften, Wiesbaden, S 309-334

Lampert T, Kroll LE (2014) Soziale Unterschiede in der Mortalität und Lebenserwartung. GBE kompakt 5 (2)

Lampert T, Kroll LE, Dunkelberg A (2007) Soziale Ungleichheit der Lebenserwartung in Deutschland. Aus Politik und Zeitgeschichte 42: 11-18

Lampert T, Kroll LE, Kuntz B et al. (2013a) Gesundheitliche Ungleichheit. In: Statistisches Bundesamt (Destatis), Wissenschaftszentrum Berlin für Sozialforschung (WZB) Bundeszentrale für politische Bildung (Hrsg) Datenreport 2013. Ein Sozialbericht für die Bundesrepublik Deutschland. Bundeszentrale für politische Bildung, Bonn, S 259-271

Lampert T, Kroll LE, Müters S et al. (2013b) Messung des sozioökonomischen Status in der Studie »Gesundheit in Deutschland aktuell« (GEDA). Bundesgesundheitsblatt - Gesundheitsforschung - Gesundheitsschutz 56 (1): 131-143

Lampert T, Kuntz B, KiGGS Study Group (2015) Gesund aufwachsen Welche Bedeutung kommt dem sozialen Status zu? GBE kompakt 6 (1) www.rki.de/gbe-kompakt (Stand: 07.03.2016)
Lampert T, Richter M, Schneider S et al. (2016) Soziale Ungleichheit und Gesundheit: Stand und Perspektiven der sozialepidemiologischen Forschung in Deutschland. Bundesgesundheitsblatt - Gesundheitsforschung - Gesundheitsschutz 59 (2):153-165

Lange C, Jentsch F, Allen J et al. (2015) Data Resource Profile: German Health Update (GEDA) - the health interview survey for adults in Germany. Int J Epidemiol 44 (2): 442-450

Leopold L, Engelhardt H (2011) Bildung und Gesundheitsungleichheit im Alter: Divergenz, Konvergenz oder Kontinuität? KZfSS Kölner Zeitschrift für Soziologie und Sozialpsychologie 63 (2): 207-236

Lindenberger U, Smith J, Mayer KU et al. (Hrsg) (2010) Die Berliner Altersstudie. 3., erw. Aufl. Akademie Verlag, Berlin

Luy M, Wegner-Siegmundt C, Wiedemann A et al. (2015) Life expectancy by education, income and occupation in Germany: estimations using the longitudinal survival method. Comparative Population Studies $40(4): 399-436$

Mackenbach JP, Huisman M, Andersen O et al. (2004) Inequalities in lung cancer mortality by the educational level in 10 European populations. Eur J Cancer 40 (1): 126-135

Markides KS, Machalek R (1984) Selective survival, aging and society. Arch Gerontol Geriatr 3 (3): 207-222

Marmot MG, Wilkinson RG (2006) Social determinants of health. Oxford University Press, Oxford; New York

Menning S, Hoffmann E (2009) Die Babyboomer - ein demografisches Porträt. Report Altersdaten 02/2009. Deutsches Zentrum für Altersfragen, Berlin

Menning S, Nowossadeck E, Maretzke S (2010) Regionale Aspekte der demografischen Alterung. Report Altersdaten 1-2/2010. Deutsches Zentrum für Altersfragen: Berlin

Mielck A (2000) Soziale Ungleichheit und Gesundheit. Empirische Ergebnisse, Erklärungsansätze, Interventionsmöglichkeiten. Verlag Hans Huber, Bern

Mielck A (2005) Soziale Ungleichheit und Gesundheit. Einführung in die aktuelle Diskussion. Verlag Hans Huber, Bern

Miilunpalo S, Vuori I, Oja P et al. (1997) Self-rated health status as a health measure: the predictive value of self-reported health status on the use of physician services and on mortality in the working-age population. J Clin Epidemiol 50 (5): 517-528

Müters S, Lampert T, Maschewsky-Schneider U (2005) Subjektive Gesundheit als Prädiktor für Mortalität. Gesundheitswesen $67(2)$ : 129-136

Oeppen J, Vaupel JW (2002) Broken Limits to Life Expectancy. Science 296 (5570): 1029-1031

Read S, Grundy E, Foverskov E (2016) Socio-economic position and subjective health and well-being among older people in Europe: a systematic narrative review. Aging \& mental health $20(5)$ : 529-542

Richter M, Hurrelmann K (Hrsg) (2009) Gesundheitliche Ungleichheit. Grundlagen, Probleme, Perspektiven. 2., aktualisierte Auflage. VS Verlag für Sozialwissenschaften, Wiesbaden

Scharein MG (2012) Altersstruktur, Fertilität, Mortalität und Migration - Vier Komponenten befeuern den demografischen Wandel. Bevölkerungsforschung Aktuell 33 (1): 23-24

Schmidt S, Petermann F, Brähler E (2012) Soziale Ungleichheit, psychische und körperliche Gesundheit - welchen Einfluss hat das Alter? Z Psychiatr Psychol Psychother 6o (3): 205-215 
Schöllgen I, Huxhold O, Tesch-Römer C (2010) Socioeconomic status and health in the second half of life: findings from the German Ageing Survey. Eur J Ageing 7 (1): 17-28

Schwarz K (1997) Bestimmungsgründe der Alterung einer Bevölkerung - Das deutsche Beispiel. Z Bevolkerungswiss 22: 347-359

Shaw BA, McGeever K, Vasquez E et al. (2014) Socioeconomic inequalities in health after age 50: are health risk behaviors to blame? Social Science \& Medicine (1982) 101: 52-60

Shkolnikov VM, Scholz R, Jdanov DA et al. (2008) Length of life and the pensions of five million retired German men. Eur J Public Health 18 (3): $264-269$

Siegrist J, Marmot MG (Hrsg) (2006) Social inequalities in health: new evidence and policy implications. Oxford University Press, Oxford; New York

Spoerri A, Zwahlen M, Egger M et al. (2006) Educational inequalities in life expectancy in German speaking part of Switzerland 1990-1997: Swiss National Cohort. Swiss Med Wkly 136 (9-10): 145-148

Thelen M, Scheidt-Nave C, Schaeffer D et al. (2012) Nationales Gesundheitsziel »Gesund älter werden«. Handlungsfeld II: Medizinische, psychosoziale und pflegerische Versorgung älterer Menschen. Bundesgesundheitsblatt - Gesundheitsforschung - Gesundheitsschutz 55 (8): 991-997

Unger R, Giersiepen K, Windzio M (2015) Pflegebedürftigkeit im Lebensverlauf: Der Einfluss von Familienmitgliedern und Freunden als Versorgungsstrukturen auf die funktionale Gesundheit und Pflegebedürftigkeit im häuslichen Umfeld. KZfSS Kölner Zeitschrift für Soziologie und Sozialpsychologie 67 (1): 193-215

Van der Heyden J, Van Oyen H, Berger N et al. (2015) Activity limitations predict health care expenditures in the general population in Belgium. BMC Public Health 15: 267

Van der Heyden JH, Schaap MM, Kunst AE et al. (2009) Socioeconomic inequalities in lung cancer mortality in 16 European populations. Lung Cancer $6_{3}(3): 322-330$

Vaupel JW (2010) Biodemography of human ageing. Nature 464 (7288): $536-542$

Voges W, Helmert U, Timm A et al. (2004) Soziale Einflussfaktoren von Morbidität und Mortalität: Sonderauswertung von Daten der Gmünder Ersatzkasse (GEK). Zentrum für Sozialpolitik, Bremen

von dem Knesebeck O, Bickel H, Fuchs A et al. (2015) Social inequalities in patient-reported outcomes among older multimorbid patients results of the MultiCare cohort study. Int J Equity Health 14 (1): 17

von dem Knesebeck O, Lüschen G, Cockerham WC et al. (2003) Socioeconomic status and health among the aged in the United States and Germany: A comparative cross-sectional study. Social Science \& Medicine 57 (9): 1643-1652

von dem Knesebeck O, Schäfer I (2009) Gesundheitliche Ungleichheit im höheren Lebensalter. In: Richter M, Hurrelmann K (Hrsg) Gesundheitliche Ungleichheit. VS Verlag für Sozialwissenschaften, Wiesbaden, S 253-265

von dem Knesebeck O, Vonneilich N (2009) Gesundheitliche Ungleichheit im Alter. Zeitschrift fur Gerontologie und Geriatrie 42 (6): 459-464

von Gaudecker H-M, Scholz RD (2007) Differential mortality by lifetime earnings in Germany. Demographic Research 17 (4): 83-108

Wing S, Manton KG, Stallard E et al. (1985) The black/white mortality crossover: investigation in a community-based study. J Gerontol 40 (1): $78-84$

Word Health Organization (2015) World report on ageing and health. WHO, Genf

Wurm S, Schöllgen I, Tesch-Römer C (2010) Gesundheit. In: MotelKlingebiel A, Wurm S, Tesch-Römer C (Hrsg) Altern im Wandel: Befunde des Deutschen Alterssurveys (DEAS). Kohlhammer, Stuttgart, S 90-117 
Impressum

GBE kompakt

Herausgeber

Robert Koch-Institut

Nordufer 20

13353 Berlin

Redaktion

Dr. Livia Ryl

Robert Koch-Institut

Abt. Epidemiologie und Gesundheitsmonitoring

General-Pape-Straße 62

12101 Berlin

Tel.: 030-18 754-3400

E-Mail: gbe@rki.de

www.rki.de/gesundheitsberichterstattung

Zitierweise

Lampert T, Hoebel J, Kuntz B, Fuchs J,

Scheidt-Nave C, Nowossadeck E (2016)

Gesundheitliche Ungleichheit im höheren Lebensalter. Hrsg. Robert Koch-Institut, Berlin.

GBE kompakt $7(1)$

www.rki.de/gbe-kompakt (Stand: o8.03.2016)

ISSN 2191-4974

DOI 10.17886 / RKI-GBE-2016-006

Das Robert Koch-Institut ist ein Bundesinstitut im Geschäftsbereich des Bundesministeriums für Gesundheit 